\title{
Erratum to: Factors affecting the adoption of e-learning systems in Qatar and USA: Extending the Unified Theory of Acceptance and Use of Technology 2 (UTAUT2)
}

\author{
Mazen El-Masri ${ }^{1} \cdot$ Ali Tarhini ${ }^{2}$ D
}

Published online: 13 April 2017

(C) Association for Educational Communications and Technology 2017

\section{Erratum to: Education Tech Research Dev DOI 10.1007/s11423-016-9508-8}

After publication of this article (El-Masri and Tarhini 2017), the editor asked us to provide additional details about this paper including why some of the hypotheses are similar to those of three previously published manuscripts (two papers and one thesis). This report will answer all questions raised related to the theoretical framework and obtained results.

The three previous studies (Tarhini et al. 2014; Tarhini et al. 2016a, b; Tarhini et al. 2017) share some of the hypotheses that have been used in this study. However, the model and the context are different. In our previous studies, we employed the technology acceptance model (Davis 1989) with a set of individual differences (age, gender, educational level) as moderators. The proposed model was then tested in the context of developing countries as exemplified by Lebanon.

The main objectives of the first study, Tarhini et al. (2014), were to empirically investigate the factors that affect the acceptance and use of e-learning in Lebanon and to investigate the role of a set of individual differences as moderators (age, gender, experience, educational level) in an extended Technology Acceptance Model (TAM). The main constructs were perceived usefulness (PU), perceived ease of use (PEOU), subjective norms (SN), and quality of work life (QWL) as well as a set of moderators (age, gender,

The online version of the original article can be found under doi:10.1007/s11423-016-9508-8.

Ali Tarhini

ali.tarhini@hotmail.co.uk

Mazen El-Masri

Mazen.elmasri@qu.edu.qa

1 College of Business and Economics, Qatar University, Doha, Qatar

2 College of Economics and Political Science, Department of Information Systems, Sultan Qaboos University, Muscat, Oman 
experience, educational level). To test the hypothesized research model, we collected data from 569 undergraduate and postgraduate students studying in Lebanon. The results revealed that perceived usefulness (PU), perceived ease of use (PEOU), subjective norms $(\mathrm{SN})$, and quality of work life (QWL) positively affect students' behavioural intention (BI). We also found that experience moderates the relationship among PEOU, PU and SN on e-learning use intention, and that age difference moderates the effects of PEOU, SN and QWL on BI. In addition, educational level moderates the effects of PEOU, SN on BI, and gender moderates the effects of PU, SN and QWL on BI. Contrary to expectations, a moderating role of age on the relationship between PU and BI was not found. Similarly, gender was not found to affect the relationship between PEOU and BI, and educational level did not moderate the relationship between PU or QWL and BI.

The main aim of the second paper, Tarhini et al. (2016b), was to develop an amalgamated conceptual model of technology acceptance that explains how individual, social, cultural, and organizational factors affect students' acceptance and usage behaviour of Web-based learning systems. More specifically, the proposed model extended the Technology Acceptance Model (TAM) to include four constructs: quality of work life, social norms, facilitating conditions and self-efficacy. In addition, individual differencesnamely, age, gender, and experience-are hypothesized to moderate the effects of these constructs on behavioural intention and technology use. The aim of the paper was only to offer opportunities for future research in understanding the acceptance of technology. To conclude, the proposed model was not tested.

In this paper (El-Masri and Tarhini 2017), we used extended Unified Theory of Acceptance and Use of Technology 2 (UTAUT2) with Trust as an external variable to examine the factors that may influence the adoption of e-learning by university students. The proposed model included the following constructs: effort expectancy, performance expectancy, price value, habit, social influence, facilitating conditions, hedonic motivation and trust. Then the proposed model was tested in the context of a developing country (Qatar) as well as a developed country (USA). There was no previous study that examined the adoption of e- learning in Qatar and the USA. The results showed that performance expectancy, hedonic motivation, habit and trust are significant predictors of behavioural intention (BI) in both samples. Contrary to our expectation, the relationship between price value and BI is insignificant. Our results also show that effort expectancy and social influence led to an increase in students' adoption of e-learning systems in developing countries but not in developed countries. Moreover, facilitating conditions increase e-learning adoption in developed countries which is not the case in developing countries.

It should be noted that the TAM (Davis 1989) and UTAUT (Venkatesh et al. 2003) are the most cited models in the history of technology adoption and diffusion. Having mentioned so, testing some of the hypotheses related to UTAUT in the context of e- learning is not new. However, extending and testing the model and its proposed hypotheses in Qatar and the USA is different. In this regard, Venkatesh et al. (2012) emphasize the importance of testing UTAUT2 in different cultures and settings in order to enhance its applicability and robustness as they argue that factors affecting the adoption of a new information system are likely to vary in context, target users and technology. Hence, this study takes into consideration the lack of cross-cultural studies between Qatar and the USA and fills a gap by extending the UTAUT2. The results of some of the hypotheses are consistent with the findings of some previous research (e.g. Venkatesh et al. 2003); however the strengths of each variable and the order of their influencing power on behavioural intention are different. 


\section{References}

Davis, F. D. (1989). Perceived usefulness, perceived ease of use, and user acceptance of information technology. MIS quarterly, 13(3), 319-340.

Iskander, M. (Ed.). (2008). Innovative techniques in instruction technology, e-learning, e- assessment and education. Springer Science \& Business Media.

Tarhini, A., Hone, K., \& Liu, X. (2014). The effects of individual differences on e-learning users' behaviour in developing countries: A structural equation model. Computers in Human Behavior, 41, 153-163.

Tarhini, A., Mohammed, A. B., \& Maqableh, M. (2016a). Modeling Factors Affecting Student's Usage Behaviour of E-Learning Systems in Lebanon. International Journal of Business and Management, 11(2), 299-312.

Tarhini, A., Elyas, T., Akour, M. A., \& Al-Salti, Z. (2016b). Technology, Demographic Characteristics and E-Learning Acceptance: A Conceptual Model Based on Extended Technology Acceptance Model. Higher Education Studies, 6(3), 72-89.

Tella, A. (2012). System-related Factors that Predict Students' Satisfaction with the Blackboard Learning System at the University of Botswana. African Journal of Library Archives and Information Science, 22(2), 41-52.

Venkatesh, V., Morris, M. G., Davis, G. B., \& Davis, F. D. (2003). User acceptance of information technology: Toward a unified view. MIS Quarterly, 27(3), 425-478.

Venkatesh, V., Thong, J., \& Xu, X. (2012). Consumer acceptance and use of information technology: Extending the unified theory of acceptance and use of technology. MIS Quarterly, 36(1), 157-178. 